This document is the Accepted Manuscript version of the following article: Martin G. H. Krause, 'Large scale simulations of the Jet-lgm Interaction', Astrophysics and Space Science, Vol. 293 (1): 255-262, August 2004. The version of record is available online at doi:

10.1023/B:ASTR.0000044674.57913.c9.

Published by Kluwer Academic Publishers 


\title{
Large scale simulations of the jet-IGM interaction
}

\author{
Martin G. H. Krause \\ Landessternwarte Königstuhl, 69117 Heidelberg, Germany
}

December 15, 2003

\begin{abstract}
In a parameter study extending to jet densities of $10^{-5}$ times the ambient one, I have recently shown that light large scale jets start their lives in a spherical bow shock phase. This allows an easy description of the sideways bow shock propagation in that phase. Here, I present new, bipolar, simulations of very light jets in $2.5 \mathrm{D}$ and $3 \mathrm{D}$, reaching the observationally relevant scale of $>200$ jet radii. Deviations from the early bow shock propagation law are expected because of various effects. The net effect is, however, shown to remain small. I calculate the X-ray appearance of the shocked cluster gas and compare it to Cygnus A and 3C 317. Rings, bright spots and enhancements inside the radio cocoon may be explained.
\end{abstract}

Keywords: extragalactic jets, jet-IGM interaction, hydrodynamics, simulations, very light jets

\section{Introduction}

X-ray studies of galaxy cluster centers containing a radio jet have shown that the jets have a considerable impact on the cluster gas (Carilli et al., 1994; Carilli et al., 2002; Blanton et al., 2001; Smith et al., 2002), forming rings, apparent spirals, and aligned features. Such systems have been claimed to be associated with very light jets (Clarke et al., 1997; Rosen et al., 1999; Krause and Camenzind, 2003). Parameter studies of very light jets have been carried out recently (Carvalho and O'Dea, 2002a; Carvalho and O'Dea, 2002b; Saxton et al., 2002; Krause, 2003; Zanni et al., 2003). There, it has been shown that very light jets first form spherically symmetric bow shocks (Krause, 2003). In that phase, they follow the expansion law (derived for a strong bow shock, which is applicable here):

$$
\int_{0}^{r} \mathcal{M}\left(r^{\prime}\right) r^{\prime} \mathrm{d} r^{\prime}=2 \int_{0}^{t} \mathrm{~d} t^{\prime} \int_{0}^{t^{\prime}} E\left(t^{\prime \prime}\right) \mathrm{d} t^{\prime \prime}
$$

where $\mathcal{M}(r)$ is an arbitrary spherically symmetric ambient gas mass distribution and $E(t)$ is the energy injection law. Here, I show $3 \mathrm{D}$ and 2.5D large scale simulations, updating the X-ray appearance of the shocked IGM, and exploring the accuracy of the spherical expansion law for the bow shock at late times.

(C) 2016 Kluwer Academic Publishers. Printed in the Netherlands. 


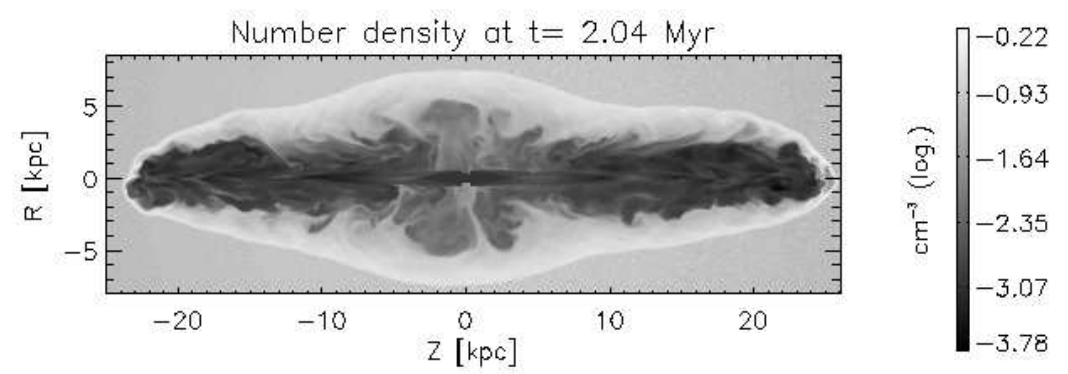

Figure 1. Logarithm of number density (slice) for the 3D run at 2.04 Myr.

\section{3D Simulation}

\subsection{Simulation Setup}

A cylindrical grid was used for the jet simulation (compare Krause and Camenzind (2002)). The size of the computational domain was: $Z \in[-69 \mathrm{kpc}, 69 \mathrm{kpc}], R \in[0,57 \mathrm{kpc}]$ and $\phi \in[0,2 \pi] .2042,805$, and 57 grid points were used in the $\mathrm{Z}, \mathrm{R}$ and $\phi$ directions, respectively. The full extend was not reached because of long computation times and breakdown of the supercomputer. With a jet radius of $r_{\mathrm{j}}=0.55$ $\mathrm{kpc}$, this gives a resolution of 8 points per beam radius (ppb). The grid was initialised with an isothermal King cluster atmosphere: $\rho_{\mathrm{e}}(r)=$ $\rho_{\mathrm{e}, 0}\left(1+\frac{r^{2}}{a^{2}}\right)^{-3 \beta / 2}$, where $r=\sqrt{R^{2}+Z^{2}}$ denotes the spherical radius, $\rho_{\mathrm{e}, 0}=1.2 \times 10^{-25} \mathrm{~g} / \mathrm{cm}^{3}$ is the characteristic density, $\beta=0.75$ and $a=35 \mathrm{kpc}$ is the core radius. In order to break the bipolar and axial symmetry, this density profile was modified by random perturbations. The jet is injected in the middle of the grid in the region $Z \in[-0.55,0.55 \mathrm{kpc}], R \in[0,0.55 \mathrm{kpc}]$, and $\phi \in[0,2 \pi]$. This region has the constant values: $\rho_{\text {jet }}=6.68 \times 10^{-28} \mathrm{~g} / \mathrm{cm}^{3}, v_{Z}= \pm 0.4 c, c$ being the speed of light. The kinetic jet luminosity is $L_{\mathrm{kin}}=1.04 \times 10^{46} \mathrm{erg} / \mathrm{s}$ for both jets together. The pressure was set in order to match the external pressure at that position. This gives a slightly varying density contrast across the grid of $\eta=\rho_{\text {jet }} / \rho_{\text {ext }} \approx 7 \times 10^{-3}$ and an internal Mach number $M=10$. The temperature in the external medium is set to $3 \times 10^{7} \mathrm{~K}$. The cooling time in the shocked cluster gas is approximately 100 Myr. The jet is expected to propagate through the whole volume in 10 Myr. So, cooling by bremsstrahlung marginally influences the state of the gas. This was taken into account. In order to keep the system in hydrostatic equilibrium, gravity by an assumed dark matter 


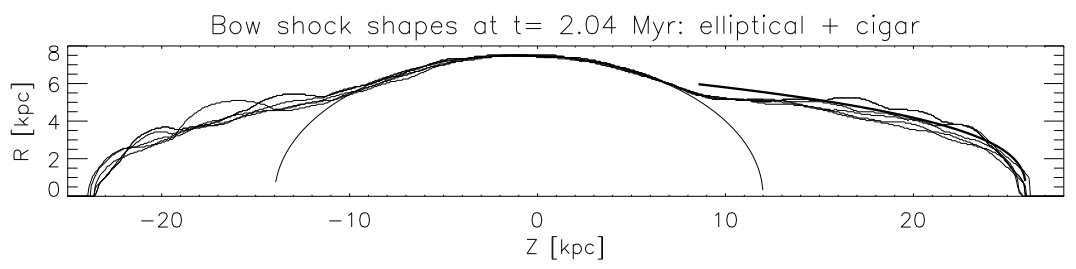

Figure 2. Bow shock shapes for the 3D run, compared to ellipse and parabola.

distribution had to be applied. The non-relativistic code NIRVANA (?) was used for the computation.

\subsection{Results}

The final snapshot at $t=2.04 \mathrm{Myr}$ is shown in Fig. 1. The cocoon is nicely placed around the jet beam. Shear instabilities show up prominently. They still grow towards the center, and develop into long fingers at the innermost positions. The pressure shows a regular spacing of shock compressed and rarefaction zones in the beam. High pressure regions are small and show up only at the end of the beams, where the Mach disk is located. The central region, with a diameter of roughly $10 \mathrm{kpc}$, is now dynamically calm. No large Mach numbers are observed there, and the pressure is approximately constant.

\subsubsection{The shape of the bow shock}

Figure 2 shows the bow shock shape for the final snapshot in detail. It has an axisymmetric part in the middle, where it can be well represented by an ellipse. The elliptical shape ends at $|Z| \approx 10$ where two cigar like extensions join the bow shock. They are 3D in nature, and can be represented, on average, by a parabola of rank three.

\subsubsection{Emission maps}

The emission due to bremsstrahlung was integrated for different viewing angles (see Fig. 3). The general X-ray emission properties of shocked ambient gas have been discussed by Clarke et al. (1997), which has been updated recently by Zanni et al. (2003). The idea is that the gas is pushed aside by the jet cocoon. Depending on its compression, it may form X-ray deficits at the location of the cocoon, and bright shells at the edges. The critical parameter is the relative shell thickness, $\xi$, defined as the width of the shocked ambient gas region divided by the local bow shock radius. X-ray deficits could be observed for $\xi>38 \%$, for sources at inclination $i=90^{\circ}$. Here, $\xi$ is comparatively low. Hence, 

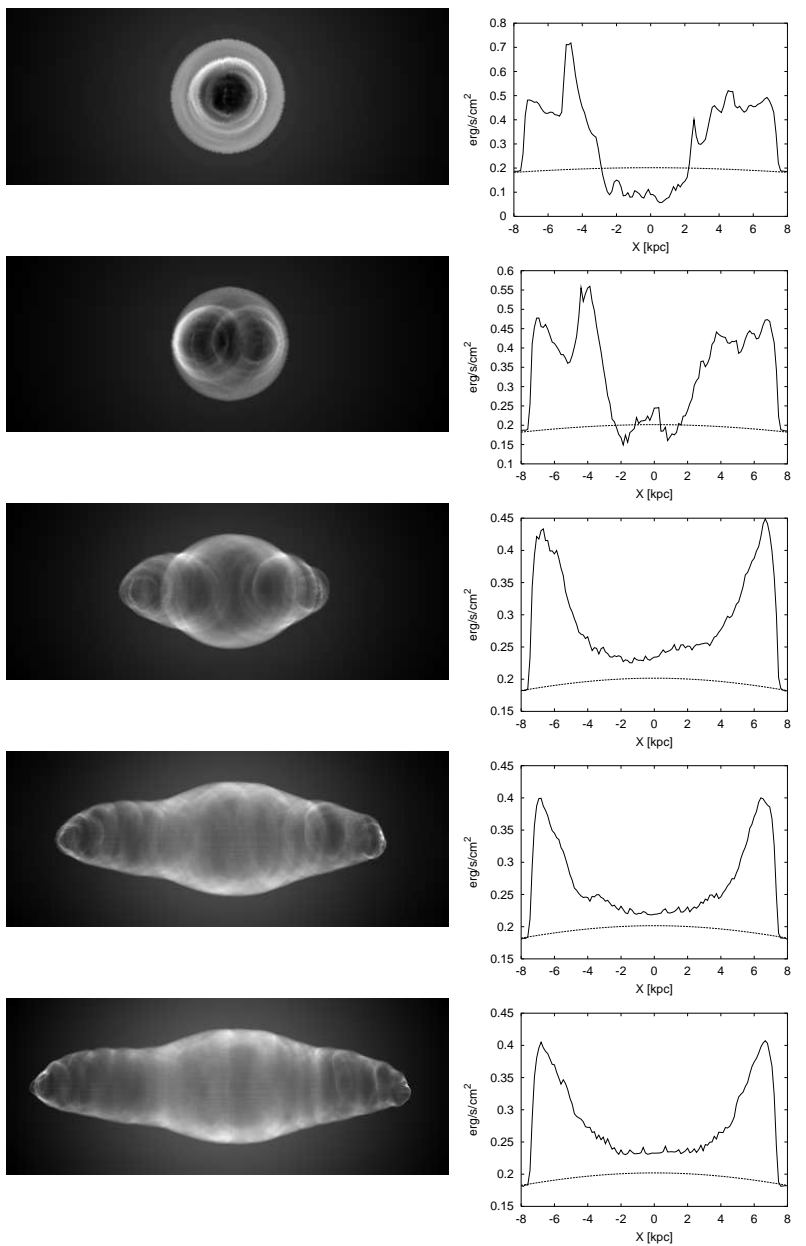
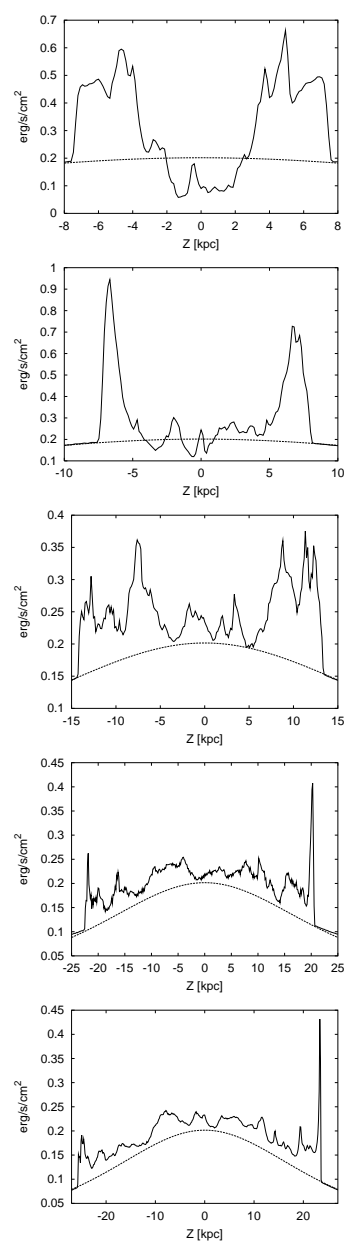

Figure 3. Bremsstrahlung emission maps for the 3D run at $t=2.04$ Myr. From top to bottom, the viewing angle is $0^{\circ}, 10^{\circ}, 30^{\circ}, 60^{\circ}$, and $90^{\circ}$. The left column shows the emission map, the middle one a vertical, and the right one a horizontal slice through the center. The undisturbed emission is indicated.

at high $i$ the $\mathrm{X}$-ray surface brightness never falls below that of the undisturbed King atmosphere, but the deficit is pronounced for low $i$ (compare Fig. 3).

The two phases of the bow shock, cigar and elliptical (see sect. 2.2.1), show up prominently in the emission maps. They form circular and elliptical rings, depending on the viewing angle. Where the rings partially overlap, they are brighter, producing the impression of ring segments (e.g. Fig. $3,10^{\circ}$ ). The structures can also intersect on the line of sight, producing bright spots (Fig. $3,30^{\circ}$ ). The pole on figures show at least 
two rings: one from the cigar phase and one from the inner elliptical part.

\section{2.5D simulation}

\subsection{Simulation Setup}

In order to study the jet evolution on larger scale, an axysymmetric (2.5D) simulation was performed with initial conditions similar to the $3 \mathrm{D}$ simulation in the previous section. The $20 \mathrm{ppb}$ simulation was run for 20 Myrs of simulation time. During that time the jet reached an extent of $110 \mathrm{kpc}$ which corresponds to 220 jet radii. The King atmosphere parameters are: $\rho_{\mathrm{e}, 0}=m_{\mathrm{p}} / \mathrm{cm}^{-3}, a=10 \mathrm{kpc}, \beta=0.75$, and $T=3 \times 10^{7} \mathrm{~K}$. The jet is injected with a density of $\rho_{\text {jet }}=10^{-4} \times \rho_{\mathrm{e}, 0}$, the sound speed in the jet was set to $0.2 c$, and the jet's Mach number to $M=3$.

\subsection{Results}

Logarithmic density and integrated X-ray emission after 20 Myr are presented in Fig. 4. Krause (2003) could only reach an evolution to up to 5 Myr. In this early phase, the bow shock is spherical, its radius following an expansion law given by (1). At later times the cocoon transforms via a conical phase towards a cylindrical one (Fig. 4).

\subsubsection{Pressure evolution}

The average jet pressure is the driving force of the inner elliptically shaped part of the bow shock. The pressure in the jet system monotonically decreases with radius. Close to the axis, the pressure is higher because of shocks in the beam region. In the shocked ambient gas region, a new equilibrium of gravity and pressure appears. The smallest pressure values are located at the bow shock, roughly $20 \%$ below the average.

It has been found that the sideways expansion of the bow shock follows the blastwave's equation of motion (Krause, 2003), in the spherical phase. The accuracy of this law will be checked in the following also for the larger 2.5D simulation. In this case, the bow shock has propagated

more than three core radii in the sideways direction. In the spherical approximation, the average pressure inside of the bow shock is given by (neglecting energy stored in the beam):

$$
p_{\mathrm{j}}=(\gamma-1)\left(L t-\mathcal{M} v^{2} / 2\right) / V_{\mathrm{j}},
$$



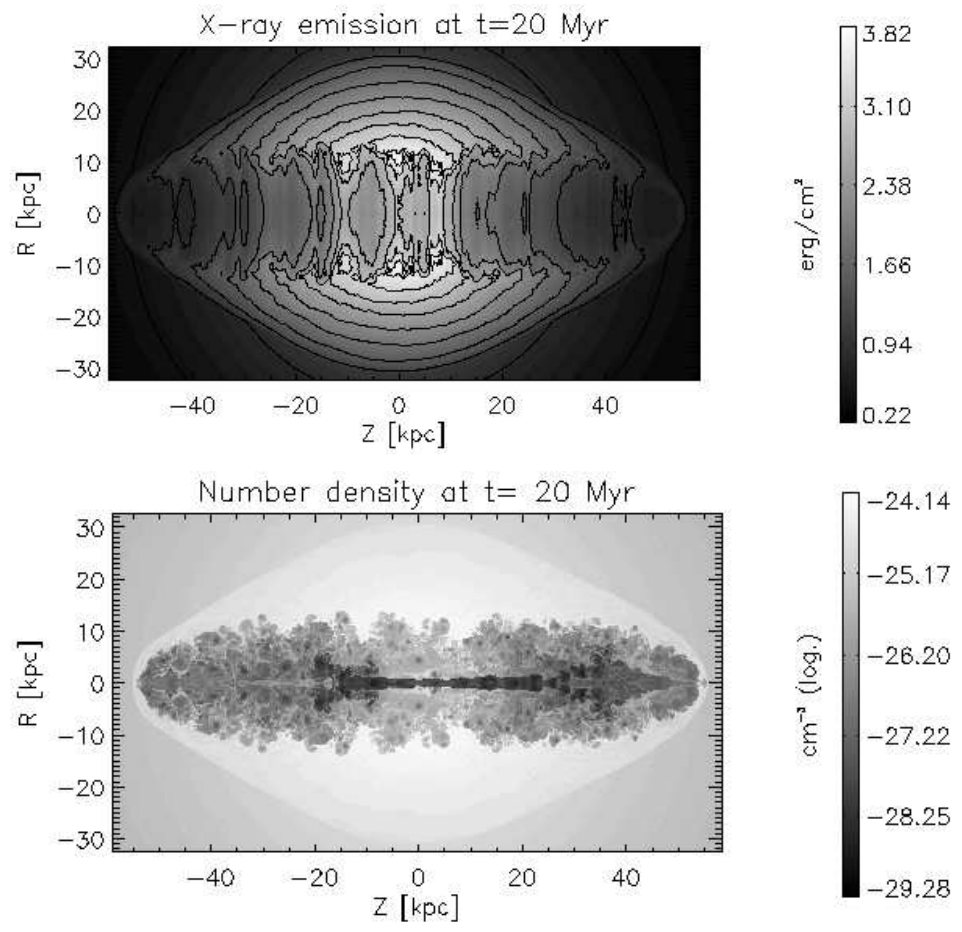

Figure 4. Final snapshots of the 2.5D simulation. Top: Line-of-sight integrated X-ray emission due to bremsstrahlung. Bottom: Logarithm of the number density.

where $V_{\mathrm{j}}$ is the jet volume (everything inside the bow shock). The power $\mathrm{L}$ includes all sources of energy, i.e. the flux of kinetic and internal energy through the jet channel, the flux of internal energy entering through the surface of the bow shock, and the energy lost by work against the gravitational field. Then, $(2)$ can be evaluated, where $v$ and $t$ are given by (1):

$$
v=\frac{L t^{2}}{\mathcal{M} r}, t=\left(\frac{3}{L} \int_{0}^{r} \mathcal{M}\left(r^{\prime}\right) r^{\prime} \mathrm{d} r^{\prime}\right)^{1 / 3} .
$$

Figure 5 shows this analytical estimate together with the data from the simulation. Here, $r$ was related to time via measurement from the simulation. The agreement is quite good, in general. The analytical formula follows the slope of the simulation data, but underestimates it by up to $\approx 20 \%$. 
Table I. Fit parameters for the bow shock position in the 2.5D simulation. The star denotes a fit with fixed $a=0$. Two stars denote fits to the spherical approximation with fixed $a=0$.

\begin{tabular}{lccclccc}
\hline time range $[\mathrm{Myr}]$ & $\mathrm{a}$ & $\mathrm{b}$ & $\mathrm{c}$ & time range $[\mathrm{Myr}]$ & $\mathrm{a}$ & $\mathrm{b}$ & $\mathrm{c}$ \\
\hline$[0: 5]$ & 2.00 & 2.67 & 0.76 & & & & \\
{$[0: 5]^{*}$} & 0 & 4.66 & 0.35 & {$[15: 20]^{*}$} & 0 & 2.99 & 0.78 \\
{$[0: 5]^{* *}$} & 0 & 3.22 & 0.67 & {$[15: 20]^{* *}$} & 0 & 2.36 & 0.81 \\
\hline
\end{tabular}

\subsubsection{Sideways motion of the inner bow shock part}

The bow shock propagation was locally fitted by a function of type $a+b t^{c}$. The resulting parameters for the different regions are shown in Table I. Usually, $a=0$ (fixed), since only the late evolution of the jet is studied. Only for the time span up to $5 \mathrm{Myr}$ a fit with $a \neq 0$ has been included because here it is possible that effects from the initial conditions still dominate the propagation. For comparison, also fits to the detailed spherical approximation are given, computed by application of (3). According to that, the exponent for the first five million years should be 0.67 . Using the pure power law, an exponent of 0.35 is achieved in the simulation data. Allowing for the radial offset gives a best fit exponent of 0.76 . Since the exponent of 0.35 is much below any expectation, it follows that the initial conditions are still important in that phase, and the fit with offset is more appropriate. The concurrence of the curves increases with time. For the last five million years, the exponent for the spherical approximation (0.81) exceeds the one for the power law fit of the simulation data (0.78). From the increasing aspect ratio, an exponent lower than the one of the spherical approximation was expected. The simulation shows that the effect is small.

\section{Discussion}

The bipolar simulations in connection with detailed studies of the early lives of very light jets reveal two parts of the bow shock: an inner elliptical part, and an outer cigar shaped one. These parts also appear in the X-ray emission. When viewed from an appropriate angle, they can appear as partial rings and bright spots. I suggest that the two X-ray rings in 3C 317 (Blanton et al., 2001) are caused by this effect.

The X-ray appearance of the $2.5 \mathrm{D}$ simulation reproduces some important details of Cygnus A's X-ray emission (Smith et al., 2002): the elliptical deformation in the shocked ambient gas region, the bright 

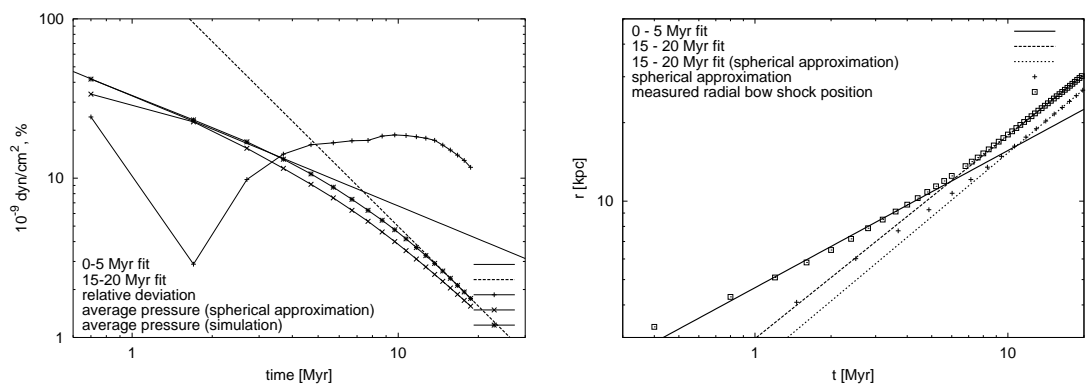

Figure 5. Left: Average jet pressure over simulation time. The stars show the values measured in the simulation, crosses mark the pressure according to a spherical approximation, plusses show the relative difference of the former in percent. Corresponding symbols are connected with solid lines. The lines show fits to the pressure measured from the simulation. The fits are: $32.84 t^{-0.69}$ (0-5 Myr, solid line), and $227.95 t^{-1.66}$ (15-20 Myr, dashed line). The best fit for the spherical approximation in the range 15-20 Myr is: $126.05 t^{-1.50}$ (not shown). Right: Bow shock radius at $\mathrm{Z}=0$ versus time (squares) with fits and compared to spherical approximation, including all power sources (see text, plus-signs). The three fits are: $4.66 t^{0.53}$ (0-5 Myr), $2.99 t^{0.78}$ (15-20 Myr), $2.36 t^{0.81}$ (15-20 Myr, spherical approximation).

X-ray filaments inside the cocoon, and the fork like structure around the cocoon. The bow shock can be located at the interface, where the elliptical X-ray contours meet the spherical ones from the unaffected cluster gas. This good agreement also supports the idea of a very light, relativistic, and magnetised jet in Cygnus A.

\section{Acknowledgements}

This work was supported by the Deutsche Forschungsgemeinschaft (Sonderforschungsbereich 439). I thank the Höchstleistungsrechenzentrum Stuttgart for super-computing time.

\section{References}

Blanton, E. L., C. L. Sarazin, B. R. McNamara, and M. W. Wise: 2001, 'Chandra Observation of the Radio Source/X-Ray Gas Interaction in the Cooling Flow Cluster Abell 2052'. ApJ 558, L15-L18.

Carilli, C. L., D. E. Harris, L. Pentericci, H. J. A. Röttgering, G. K. Miley, J. D. Kurk, and W. van Breugel: 2002, 'The X-ray - radio alignment in the $\mathrm{z}=2.2$ radio galaxy PKS 1138-262'. ApJ.

Carilli, C. L., R. A. Perley, and D. E. Harris: 1994, 'Observations of Interaction Between Cluster Gas and the Radio Lobes of Cygnus-A'. MNRAS 270, 173+. 
Carvalho, J. C. and C. P. O'Dea: 2002a, 'Evolution of Global Properties of Powerful Radio Sources. I. Hydrodynamical Simulations in a Constant Density Atmosphere and Comparison with Self-similar Models'. ApJS 141, 337-370.

Carvalho, J. C. and C. P. O'Dea: 2002b, 'Evolution of Global Properties of Powerful Radio Sources. II. Hydrodynamical Simulations in a Declining Density Atmosphere and Source Energetics'. ApJS 141, 371-414.

Clarke, D. A., D. E. Harris, and C. L. Carilli: 1997, 'Formation of cavities in the X-ray emitting cluster gas of Cygnus A'. MNRAS 284, 981-993.

Krause, M.: 2003, 'Very light jets. I. Axisymmetric parameter study and analytic approximation'. $A \mathscr{E} A$ 398, 113-125.

Krause, M. and M. Camenzind: 2002, 'A 3D Hydrodynamic Simulation for the Cygnus A Jet as a Prototype for High Redshift Radio Galaxies'. In: High Performance Computing in Science and Engeneering '01, eds.: Krause, E. and Jäger, W., Springer. pp. 329+.

Krause, M. and M. Camenzind: 2003, 'Parameters for very light jets of cD galaxies'. New Astronomy Review 47, 573-576.

Rosen, A., P. A. Hughes, G. C. Duncan, and P. E. Hardee: 1999, 'A Comparison of the Morphology and Stability of Relativistic and Nonrelativistic Jets'. ApJ 516, 729-743.

Saxton, C. J., R. S. Sutherland, G. V. Bicknell, G. F. Blanchet, and S. J. Wagner: 2002, 'Complex shock structure in the western hot-spot of Pictor A'. A $\& A$ 393, $765-792$.

Smith, D. A., A. S. Wilson, K. A. Arnaud, Y. Terashima, and A. J. Young: 2002, 'A Chandra X-ray Study of Cygnus A - III. The Cluster of Galaxies'. ApJ $56 \mathbf{6 5 .}$

Zanni, C., G. Bodo, P. Rossi, S. Massaglia, A. Durbala, and A. Ferrari: 2003, 'X-ray emission from expanding cocoons'. A\&A 402, 949-962. 
krause.tex; 15/11/2016; 7:37; p.10 